\title{
An Adaptive Growing and Pruning Algorithm for Designing Recurrent Neural Network
}

\author{
Hong-Gui Han*, and Jun-Fei Qiao
}

\begin{abstract}
The training of recurrent neural networks (RNNs) concerns the selection of their structures and the connection weights. To efficiently enhance generalization capabilities of RNNs, a recurrent self-organizing neural networks (RSONN), using an adaptive growing and pruning algorithm (AGPA), is proposed for improving their performance in this paper. This AGPA can self-organize the structures of RNNs based on the information processing ability and competitiveness of hidden neurons in the learning process. Then, the hidden neurons of RSONN can be added or pruned to improve the generalization performance. Furthermore, an adaptive second-order algorithm with adaptive learning rate is employed to adjust the parameters of RSONN. And the convergence of RSONN is given to show the computational efficiency. To demonstrate the merits of RSONN for data modeling, several benchmark datasets and a real world application associated with nonlinear systems modeling problems are examined with comparisons against other existing methods. Experimental results show that the proposed RSONN effectively simplifies the network structure and performs better than some exiting methods.
\end{abstract}

Index Terms-Recurrent self-organizing neural network; adaptive growing and pruning algorithm; information processing ability; competitiveness; convergence.

\section{INTRODUCTION}

$\mathrm{I}$ connection weights between the neurons. The input neurons are set by the environment and the output neurons are computed using the connection weights and the hidden neurons [1-2]. The states of hidden neurons can store information through time. In principle, an RNN is a powerful tool that has been successfully employed in many applications such as system identification and control [3-4], time series prediction [5-6], and some other engineering areas [7-8]. However, RNNs have been proved to be difficult to choose a suitable structure for a particular application [9-10].

In fact, if the structures of RNNs are too large, the networks tend to overfitting and poor generalization. While if the structures of RNNs are too small, the networks may not be good enough to achieve the desired performance [11-12]. In general, to design the structures of RNNs for improving their generalization performance, there are three ways: constructive algorithm, 
pruning algorithm and constructive-and-pruning algorithm [13-15]. For example, Juang et al. proposed a constructive algorithm for organizing RNNs based on a clustering algorithm in [16]. The results demonstrated that this proposed constructive RNN can be used for the nonlinear system prediction and identification. And a constructive Elman-type RNN, based on a combination of particle swarm optimization and covariance matrix adaptation algorithm, was developed in [17]. Additionally, some other constructive algorithms for RNNs have been already proposed in [18-19]. These constructive algorithms can add hidden neurons for a minimal RNN. Then, the new hidden neurons are incrementally changed until pre-specified error requirement is reached. However, the constructive algorithms tend to build small networks due to their incremental learning nature cannot prune the redundant hidden neurons [20]. Moreover, when to stop the addition of hidden neurons is still a hurdle that constructive algorithms need to overcome. Unlike the constructive algorithms, the pruning algorithms start with an over-sized structure and deletes the unnecessary hidden neurons in the training process. Mirikitani et al. proposed a pruning strategy, based on the recursive Bayesian Levenberg-Marquardt algorithm, to evaluate the significance of hidden neurons to prune the redundant hidden neurons of RNNs [21]. Experimental results demonstrated that the proposed approach outperforms standard real-time recurrent learning and extended Kalman training algorithms. Yin et al. introduced a developmental approach to prune the reservoir computing model by a gene regulatory network mechanism in [22]. The results on both the benchmark and real-world problems revealed that the proposed algorithm was effective and robust. And in [23], a recursive least square learning algorithm was proposed to train and prune a recurrent radial basis function network. Moreover, some other pruning algorithms for RNNs have been proposed in [24-25]. Recent interest has been tend on the pruning algorithms. However, the computational cost of the pruning algorithms is higher since the majority of the training time is spent on networks larger than necessary. And it is difficult to decide when to stop searching for a smaller version of RNNs [26].

Recently, the constructive-and-pruning algorithms, executing a constructive phase first and then a pruning phase, have been used to solve some of the above problems [27-28]. Hsu et al. developed a self-constructing RNN (SCRNN) to mimic an ideal computation controller [29]. The results demonstrated that this SCRNN owned high learning ability and low computation complexity. Juang et al. introduced a recurrent self-evolving fuzzy neural network with local feedbacks (RSEFNN-LF) for modeling nonlinear systems. This RSEFNN-LF was constructed via simultaneous structure and parameter learning phases. The simulations results validated the advantages of the proposed RSEFNN-LF [30]. Moreover, a self-organizing recurrent-neural- fuzzy network (SORNFN) was proposed for the trajectory tracking control of linear microstepping motor drives in [31]. In SORNFN, a set of recurrent fuzzy rules can be built online through the concurrent structure and parameters learning algorithms. In addition, the constructive-and-pruning algorithms have attracted a lot of attention by many researchers 
and several promising algorithms have been proposed in the literatures [32-34]. However, few of these self-organizing RNNs considered the information processing ability which is an important issue of the biological neural system [35]. Therefore, it is still a challenging problem to design an effective algorithm for designing RNNs with guaranteed generalization performance [36-40].

Based on the above analysis, a recurrent self-organizing neural networks (RSONN), based on an adaptive growing and pruning algorithm (AGPA), is developed in this paper. The contributions of this paper are as follows: First, the AGPA, based on the information processing ability and competitiveness of hidden neurons, is used to grow and prune the hidden neurons of RSONN. Second, the structure of RSONN can be self-organized systematically to reduce the computational complexity and improve the generalization performance. Third, the convergence of RSONN has been demonstrated theoretically and experimentally. Moreover, the theoretical analysis provides some sufficient conditions for the convergence.

The rest of this paper is structured as follows. Section II describes the structure of RNN. The AGPA as well as the structure learning and parameter learning algorithms of RSONN are described in Section III. The convergence of RSONN is discussed in Section IV. Then, the experimental results are presented to demonstrate the superior performances of RSONN in section V. Finally, the conclusion is given in section VI.

\section{Structure OF RECURRENT NeURAL Network (RNN)}

In this section, the structure of RNN is introduced as shown in Fig. 1. To give a clear understanding of the network structure, the signal propagation and the basic function in each layer of RNN are described below.

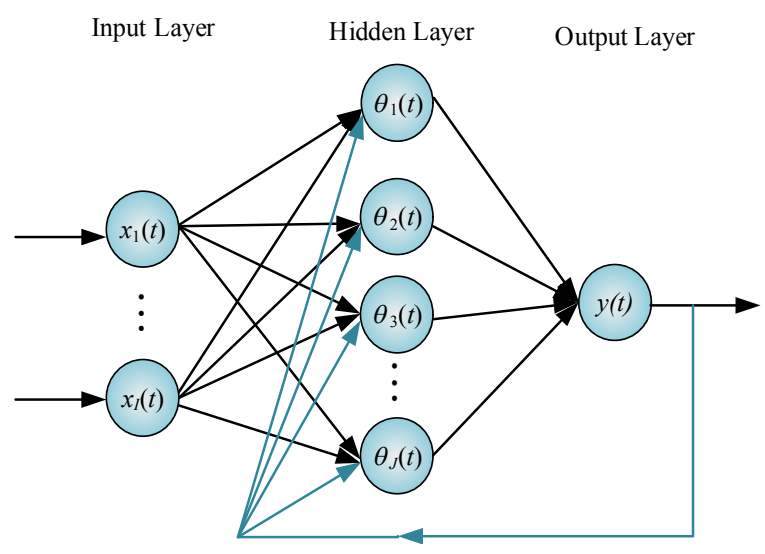

Fig. 1. The structure of RNN. ( $\mathrm{Z}^{-1}$ represents a one-time step delay unit)

Input layer: There are I neurons in this layer, and the output value of each neuron is:

$$
u_{i}(t)=x_{i}(t)
$$

where $u_{i}(t)$ is the $i$ th output value at time $t, i=1,2, \ldots, I$, and the input vector is $\mathbf{x}(t)=\left[x_{1}(t), x_{2}(t), \ldots, x_{I}(t)\right]$. 
Hidden layer: Each neuron in hidden layer connects with the input neurons and the output neuron. The outputs of hidden neurons are:

$$
\theta_{j}(t)=e^{-\left\|\mathbf{h}_{j}(t)-\mathbf{c}_{j}(t)\right\| / 2 \sigma_{j}^{2}(t)},(j=1,2, \ldots, J)
$$

where $J$ is the number of hidden neurons, $\left\|\mathbf{h}_{j}-\mathbf{c}_{j}\right\|$ represents the Eucidean distance between $\mathbf{h}_{j}$ and $\mathbf{c}_{j}$, $\mathbf{c}_{j}$ and $\sigma_{j}$ represent the center and radius of the $j$ th hidden neuron, respectively; $\mathbf{C}(t)=\left[\mathbf{c}_{1}(t), \mathbf{c}_{2}(t), \ldots, \mathbf{c}_{J}(t)\right]^{\mathrm{T}}$, and $\mathbf{h}_{j}$ is the input vector of the $j$ th hidden neuron

$$
\mathbf{h}_{j}(t)=\left[h_{j 1}(t), h_{j 2}(t), \cdots, h_{j I}(t)\right], h_{j i}(t)=u_{i}(t)+v_{j}(t) y(t-1) \text {, }
$$

where $v_{j}(t)$ is the feedback weight connecting the $j$ th hidden neuron with the output neuron, $\mathbf{v}(t)=\left[v_{1}(t), v_{2}(t), \ldots, v_{J}(t)\right]^{\mathrm{T}}$, and $y(t-1)$ is the output value of RNN at time $t-1$.

Output layer: The output value is:

$$
y(t)=\sum_{j=1}^{J} w_{j}(t) \theta_{j}(t)
$$

where $y(t)$ is the output value of output layer at time $t, w_{j}(t)$ is the $j$ th output weight, $\mathbf{w}(t)=\left[w_{1}(t), w_{2}(t), \ldots, w_{J}(t)\right]^{\mathrm{T}}$.

\section{ReCURRENT SELF-Organizing NeURAL NETwORK (RSONN)}

The performance of RNN mainly relies on its structures and the connection weights. Fig. 2 shows the block diagram of the learning processes. In this section, the structure and the parameters learning algorithms are described in details.

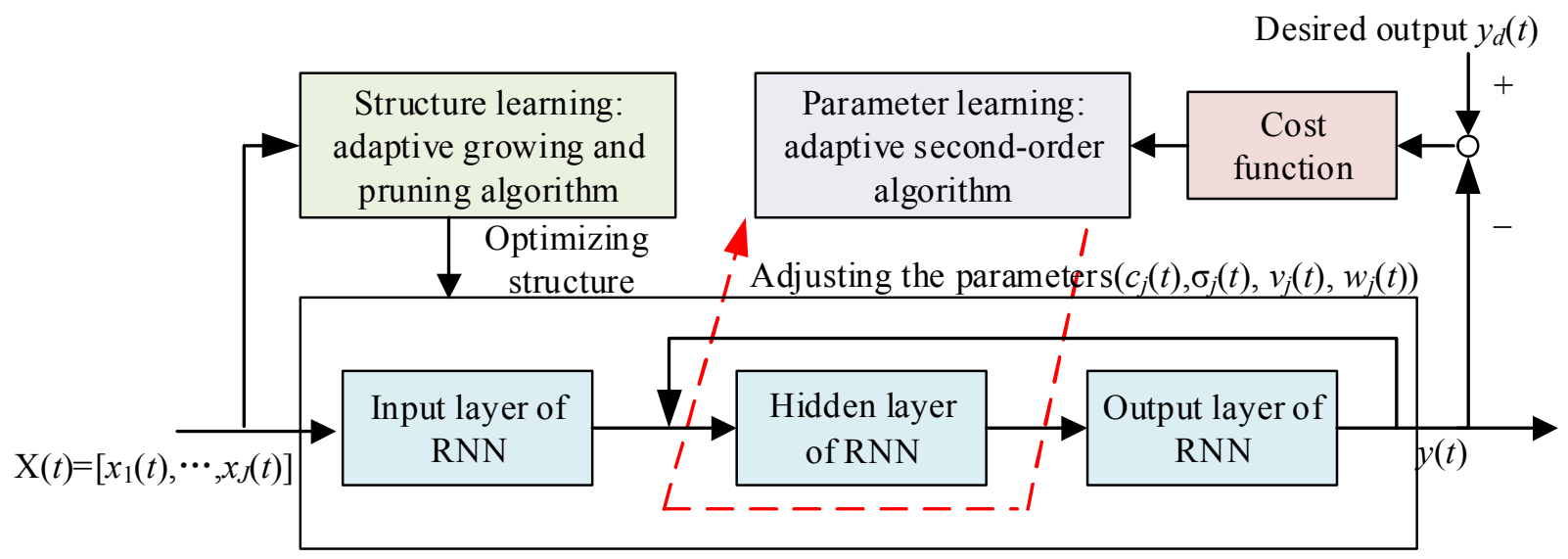

Fig. 2. The block diagram of structure and parameter learning processes for RSONN

For RSONN, the AGPA is used to design the structure of RNN by the information process ability and competitiveness of hidden neurons. Meanwhile, an adaptive second-order algorithm [41] is used to train the parameters. 


\section{A. Adaptive Growing and Pruning Algorithm (AGPA)}

In the biological neural system, the identification of active neurons is a fundamental challenge in understanding the neural basis of behavior. The neurons has a few thousand synapses. Each synapse can receive signals from other neurons, raising or lowering the electrical potential inside the neuron. Since the neurons have limited-resource setting, they are competed with each other. When the potential reaches its threshold, the neuron will fire and a pulse will be sent through the axon to other neurons. Therefore, after an overproduction of neurons and a transient exuberant number of connections followed by cell death and selective synaptic pruning, the neurons with the stronger competitiveness are suitable for survival and growth [42]. Based on the above analysis, in this paper, the proposed AGPA derives from the neural development and differentiation mechanism. Then the hidden neurons with higher competitiveness are split into new neurons. The hidden neurons with smaller competitiveness, on the other side, will be pruned to simplify the structure of RNN. The details of AGPA are described as follows:

\section{1) Growing Phase}

Based on the discussion in [42], the stronger neurons are in line with the stronger competitiveness. The competitiveness of hidden neurons can be defined as:

$$
c p_{j}(t)=\rho_{j}(t) s_{j}(t) \sigma_{j}(t),(j=1,2, \cdots, J)
$$

where $c p_{j}(t)$ is the competitiveness of the $j$ th hidden neuron, $\sigma_{j}(t)$ is the radius of the $j$ th hidden neuron, $\rho_{j}(t)$ denotes the correlation coefficient between the hidden layer output and network output, $s_{i}(t)$ is the active state of the $j$ th hidden neuron

$$
s_{j}(t)=\chi^{-\left\|\mathbf{h}_{j}(t)-\mathbf{c}_{j}(t)\right\|}
$$

where $\chi \in(1,2)$, and the correlation coefficient $\rho_{j}(t)$ is

$$
\rho_{j}(t)=\frac{\sum_{k=0}^{K}\left[A_{j}(t-k)-\bar{A}(t)\right][B(t-k)-\bar{B}(t)]}{\sqrt{\sum_{k=0}^{K}\left[A_{j}(t-k)-\bar{A}(t)\right]^{2} \sum_{k=0}^{K}[B(t-k)-\bar{B}(t)]^{2}}},
$$

where $K$ is a constant, $A_{j}(t)=\left[w_{j}(t) \theta_{j}(t), w_{j}(t-1) \theta_{j}(t-1), \ldots w_{j}(t-k) \theta_{j}(t-k), \ldots w_{j}(t-K) \theta_{j}(t-K)\right]^{\mathrm{T}}$ and $B(t)=[y(t), y(t-1), \ldots, y(t-k), \ldots$ $y(t-K)]^{\mathrm{T}}$ are the matrix of hidden neurons and output neuron, respectively; $\bar{A}(t)$ and $\bar{B}(t)$ are the means of $A_{j}(t)$ and $B(t)$. Based on Eq. (7), if the value of $\rho_{j}(t)$ is nearly equal to 1 , the $j$ th hidden neuron has large relevance to the output neuron. For RNN, when the information processing ability is insufficient in the learning process, the hidden neurons with the largest competitiveness will be split 


$$
E(t)-E(t+\tau) \leq \varepsilon, \quad j=\arg \max _{1 \leq j \leq J(t)}\left(c p_{j}(t)\right)
$$

where $E(t)$ and $E(t+\tau)$ are the training error at times $t$ and $t+\tau$ respectively, $\tau$ is an interval, and $\varepsilon$ is the pre-specified threshold, $J(t)$ is the number of existing neurons at time $t$. Then, a new neuron will be added to improve the performance. Moreover, in order to maintain convergence of RSONN, the initial parameters of the new hidden neuron are:

$$
\mathbf{c}_{J+1}(t)=\alpha \mathbf{c}_{j}(t)+\beta \mathbf{h}_{j}(t), \sigma_{J+1}(t)=\alpha \sigma_{j}(t), v_{J+1}(t)=\alpha v_{j}(t), w_{J+1}=e(t)\left(\theta_{J+1}(t)\right)^{-1}
$$

where $\mathbf{c}_{j}(t)$ and $\sigma_{j}(t)$ are the center and radius of the $j$ th hidden neuron, and $\mathbf{c}_{J+1}(t)$ and $\sigma_{J+1}(t)$ are the center and radius of the new hidden neuron, respectively; $\alpha \in[0.95,1.05]$ and $\beta \in[0,0.1], v_{J+1}(t)$ and $v_{j}(t)$ are the feedback weights of the new hidden neuron and the $j$ th neuron, respectively, $w_{J+1}(t)$ and $\theta_{J+1}(t)$ are the connection weights and the output of the new hidden neuron.

\section{2) Pruning Phase}

In this phase, the redundant neurons are removed, if

$$
c p_{j}(t)<\xi
$$

where $\zeta \in\left(0, E_{d}\right)$ is the preset pruning threshold and $E_{d}$ is the preset constant. When the $j$ th hidden neuron will be pruned, find the neuron $j$ ' in the hidden layer which has the minimal Euclidean distance between the $j$ th hidden neuron and the $j$ 'th hidden neuron. The connection weights of the $j$ 'th hidden neuron will be updated as:

$$
w_{j^{\prime}}^{\prime}(t)=w_{j^{\prime}}(t)+\frac{\theta_{j}(t)}{\theta_{j^{\prime}}(t)} w_{j}(t),
$$

where $w_{j^{\prime}}^{\prime}(t)$ and $w_{j}^{\prime}(t)$ are the connection weights between the $j$ 'th hidden neuron and the output neuron before and after the $j$ th hidden neuron is pruned, $\theta_{j^{\prime}}(t)$ and $\theta_{j}(t)$ are the outputs of the $j$ 'th and the $j$ th hidden neurons before the $j$ th hidden neuron is cut off. The center, radius, and feedback weight of the $j$ 'th hidden will be unchanged after the $j$ th hidden neuron is pruned.

Remark 1: The proposed AGPA, based on the information processing ability and competitiveness of hidden neurons, can choose the right neurons in the growing and pruning phases.

Remark 2: Simulating this biological neural system, the structure adjustment mechanism as Eq. (5), the proposed AGPA, with the competition coefficient of each neuron, can self-organize the structure of RSONN in the learning process. Moreover, the proposed method, in many cases, may also lead to the structure design of the other recurrent neural network.

\section{B. Parameter learning algorithm}

To construct RSONN with high generalization performance, the parameters (including the centers, widths and weights) of RSONN are trained by the adaptive second-order algorithm [41]. The update rule of parameters is: 


$$
\mathbf{W}(t+1)=\mathbf{W}(t)+(\boldsymbol{\Psi}(t)+\eta(t) \times \mathbf{I})^{-1} \times \mathbf{\Omega}(t),
$$

where $\mathbf{W}(t)$ is the variable vector, $\mathbf{W}(t)=[\mathbf{v}(t), \mathbf{w}(t), \mathbf{C}(t), \boldsymbol{\sigma}(t)], \mathbf{I}$ is the identify matrix that helps avoiding the ill condition in solving inverse matrix, $\eta(t)$ is the adaptive learning rate defined as:

$$
\begin{gathered}
\eta(t)=\mu(t) \eta(t-1), \\
\mu(t)=\frac{\left(\beta^{\max }(t)+\eta(t-1)\right) /\left(1+\beta^{\max }(t-1)\right)-\beta^{\min }(t-1)}{\eta(t-1)},
\end{gathered}
$$

$\beta^{\max }(t)$ and $\beta^{\min }(t)$ are the maximum and minimum eigenvalues of $\Psi(t)$, respectively; $0<\beta^{\min }(t)<\beta^{\max }(t), 0<\eta(t)<1$, in addition, the quasi Hessian matrix $\boldsymbol{\Psi}(t)$ and the gradient vector $\boldsymbol{\Omega}(t)$ are:

$$
\begin{gathered}
\boldsymbol{\Psi}(t)=\mathbf{j}^{\mathrm{T}}(t) \mathbf{j}(t), \\
\boldsymbol{\Omega}(t)=\mathbf{j}^{\mathrm{T}} e(t), \\
e(t)=y_{d}(t)-y(t),
\end{gathered}
$$

$e(t)$ is the approximation error, $y_{d}(t)$ is the desired output and $y(t)$ is the network output at time $t$, and the Jacobian vector $\mathbf{j}(t)$ is calculated as:

$$
\mathbf{j}(t)=\left[\frac{\partial e(t)}{\partial \mathbf{v}(t)}, \frac{\partial e(t)}{\partial \mathbf{w}(t)}, \frac{\partial e(t)}{\partial \mathbf{C}(t)}, \frac{\partial e(t)}{\partial \boldsymbol{\sigma}(t)}\right]
$$

In the process of parameters learning, the variable vector $\mathbf{W}(t)$ contains four kinds of variables: the center vector $\mathbf{c}(t)$, the width vector $\boldsymbol{\sigma}(t)$, the feedback weight matrix $\mathbf{v}(t)$, the output weight matrix $\mathbf{w}(t)$.

According to Eqs. (1)-(4), the elements of the Jacobian $\mathbf{j}(t)$ are given as:

$$
\begin{gathered}
\frac{\partial e(t)}{\partial c_{i j}(t)}=-\frac{w_{j}(t) \theta_{j}(t)\left(u_{i}(t)+y(t-1) v_{j}(t)-c_{i j}(t)\right)}{\sigma_{i j}^{2}(t)} \\
\frac{\partial e(t)}{\partial \sigma_{i j}(t)}=-\frac{w_{j}(t) \theta_{j}(t)\left\|u_{i}(t)+y(t-1) v_{j}(t)-c_{i j}(t)\right\|^{2}}{\sigma_{i j}^{3}(t)} \\
\frac{\partial e(t)}{\partial v_{j}(t)}=\frac{w_{j}(t) \theta_{j}(t)\left(u_{i}(t)+y(t-1) v_{j}(t)-c_{i j}(t)\right) y(t-1)}{\sigma_{i j}^{2}(t)} \\
\frac{\partial e(t)}{\partial w_{j}(t)}=-\theta_{j}(t) .
\end{gathered}
$$

With Eqs. (19)-(22), all the elements of the Jacobian vector $\mathbf{j}(t)$ can be calculated. 


\section{Recurrent Self-Organizing Neural Network (RSONN)}

In RSONN, the performance can be improved by using the AGPA and the adaptive second-order algorithm for adjusting both the structure and parameters. Based on the above analysis, the proposed RSONN learning scheme can be described by the flowchart shown in Fig.3, which can be explained further as follows.

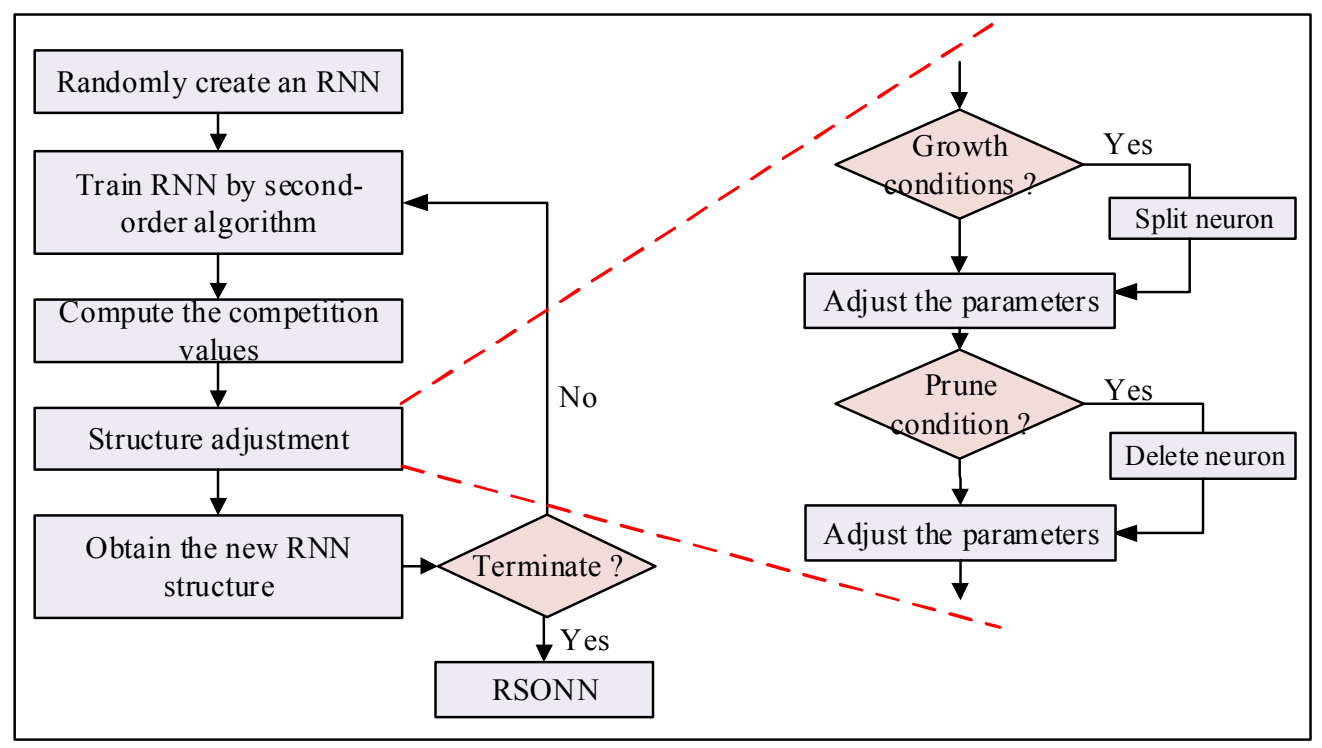

Fig. 3. Flowchart of RSONN

Step 1) Create an initial three-layer RNN. The number of neurons in the input and output layers is the same as the number of input and output variables in the problem that is being solved. The number of neurons in the hidden layer is randomly generated. The number of sample is $P$. All of the parameters should be initialized with the center, radius, feedback weights and output weights of RNN uniformly distributed within a small range.

Step 2) For the input sample $\mathbf{x}(t)$, the center, radius, feedback weights and output weights of RNN are adjusted using the adaptive second-order algorithm.

Step 3) Compute the competitiveness of hidden neurons as Eq. (5). If the condition meets the growing phase criteria, go to step 4). If the condition meets the pruning phase criteria, go to step 5). Otherwise, go to step 6).

Step 4) Split the $j$ th hidden neuron and insert new hidden neurons. The initial parameters of the new neurons can be given as Eq. (9).

Step 5) Delete the connection between the $j$ th hidden neuron and the output neuron, update the remaining parameters by Eq. (11).

Step 6) $p=p+1$, go to step 2). Stop when $p=P$. 


\section{Convergence Analysis}

In this section, the convergence of RSONN is analyzed, and we show that the convergence can be guaranteed in both the structure fixed phase and the structure updating phase.

\section{A. Structure Fixed Phase}

For the convenience of the following proof, this study chooses the Lyapunov function as:

$$
V(\mathbf{W}(t))=\frac{1}{2} e^{2}(t)
$$

the change of the Lyapunov function $V(\mathbf{W}(t))$ is obtained according to references [43]-[44]:

$$
\Delta V(\mathbf{W}(t))=V(\mathbf{W}(t+1))-V(\mathbf{W}(t))=-\nabla \mathbf{E}^{T}(\mathbf{W}(t)) \Delta \mathbf{W}(t)+\frac{1}{2} \Delta \mathbf{W}^{T}(t) \nabla^{2} \mathbf{E}(\mathbf{W}(t)) \Delta \mathbf{W}(t)
$$

where $\nabla \mathbf{E}(\Delta \mathbf{W}(t))$ and $\nabla^{2} \mathbf{E}(\Delta \mathbf{W}(t))$ are the matrix of first order and second order derivatives of the cost function, respectively. Using the update rule as defined in Eq. (12), there will be:

$$
\begin{gathered}
\Delta \mathbf{W}(t)=(\boldsymbol{\Psi}(\mathbf{W}(t))+\eta(t) \mathbf{I})^{-1} \mathbf{\Omega}(t), \\
\nabla \mathbf{E}(\mathbf{W}(t))=\mathbf{\Omega}(\mathbf{W}(t)), \\
\nabla^{2} \mathbf{E}(\mathbf{W}(t))=\mathbf{\Psi}(\mathbf{W}(t))+\eta(t) \mathbf{I} .
\end{gathered}
$$

Now we have the following convergence theorems.

Theorem 1 (Convergence of RSONN with fixed structure). Suppose the number of hidden neurons of RSONN is $J$, and the RSONN is trained by the update rule defined by Eq. (12), if

$$
\begin{gathered}
\|\Delta \mathbf{W}(t)\| \leq \kappa(t), \\
\kappa(t)=\min \left\{\|\Delta \mathbf{W}(t-1)\|, \frac{\boldsymbol{\Omega}(\mathbf{W}(t-1))}{\mathbf{\Psi}(\mathbf{W}(t-1))}\right\},
\end{gathered}
$$

then the convergence of RSONN can be guaranteed and $e(t) \rightarrow 0$ as $t \rightarrow \infty$.

Proof. Combining Eqs. (24)-(27), there holds the results:

$$
\Delta V(\mathbf{W}(t))=-\frac{1}{2} \Delta \mathbf{W}^{T}(t) \nabla^{2} \mathbf{E}(\mathbf{W}(t)) \Delta \mathbf{W}(t)
$$

When the situations (28)-(29) are satisfied, the matrix $\nabla^{2} \mathbf{E}(\Delta \mathbf{W}(t))$ is positive definite, one can further has

$$
\Delta V(\mathbf{W}(t))<0
$$

It follows from (23) and (31) that $V$ is not increasing, one can further conclude that 


$$
\lim _{t \rightarrow \infty} e(t)=0,
$$

and the convergence result of Theorem 1 is thus completed.

Remark 3: Theorem 1 is stated for RSONN with a fixed structure. However, the theorem will be investigated for RSONN with varying structure, that is, for the case when the hidden neurons change with time. The reason is that the structure change may destroy the convergence of the RSONN if the structure design method is not suitable.

\section{B. Structure Self-Organizing Phase}

The convergence of the proposed RSONN with structure self-organizing phase will be discussed in details.

Theorem 2. (Convergence of RSONN with structure self-organizing) For RSONN, if the structure is self-organized in the learning process and the adaptive second-order algorithm is designed as Eq. (12), then the convergence of the proposed RSONN can be guaranteed and $e(t) \rightarrow 0$ as $t \rightarrow \infty$.

Proof. When a new hidden neuron has been added to the network structure (there are $J+1$ hidden neurons after the growing step), the approximation error $e^{\prime}(t)$ with $J+1$ hidden neurons is given by

$$
e^{\prime}(t)=y_{d}(t)-\sum_{j=1}^{J+1} w_{j}(t) \theta_{j}(t)=y_{d}(t)-\left(\sum_{j=1}^{J} w_{j}(t) \theta_{j}(t)+w_{J+1}(t) \theta_{J+1}(t)\right) .
$$

Based on Eq. (9), one has

$$
e^{\prime}(t)=y_{d}(t)-y(t)-\frac{e(t)}{\theta_{J+1}(t)} \theta_{J+1}(t)=0
$$

Meanwhile, when a hidden neuron has been pruned in the hidden layer (there are $J-1$ hidden neurons after the pruning step), the approximation error $e^{\prime \prime}(t)$ after the neuron pruning is given by

$$
e^{\prime \prime}(t)=y_{d}(t)-\sum_{j=1}^{J-1} w_{j}(t) \theta_{j}(t)=y_{d}(t)-\sum_{j=1, j \neq j^{\prime}}^{J} w_{j}(t) \theta_{j}(t)-w_{j^{\prime}}(t) \theta_{j^{\prime}}(t) .
$$

Based on Eq. (11), it will be

$$
e^{\prime \prime}(t)=y_{d}(t)-\sum_{j=1}^{J} w_{j}(t) \theta_{j}(t)=e(t) .
$$

Eqs. (34) and (36) clearly show that the output approximation error will not be influenced after growing or pruning phases, respectively. Moreover, as can be seen in Theorem 2, the convergence of RSONN can be maintained in the structure updating phase, this means that the structure and parameters adjusting algorithms proposed in this paper can guarantee the convergence of RSONN which is one of the most serious features. 
Remark 4: Theorems 1 and 2 show that the convergence of RSONN can be maintained in both fixed structure and structure self-organizing phases. The proposed RSONN will be convergent from any starting point and converge to a stationary point of the cost function where the situations hold.

Remark 5: For the proposed method, the involved computation is dominated by the training algorithm for $\mathbf{W}$. In this remark, the computational complexity analysis is given. Suppose $\{(\mathbf{x}(t), \mathrm{y}(t)) ; t=1, \ldots, P\}$ is a set of data samples. The structure of neural network is $I-J-1$ ( $I$ input variables, $J$ hidden neurons and a single-output neuron). The computational complexity results were compared with three other algorithms: the modified Gram-Schmidt (MGS) [3], the GD [55], and the AFS [56]. Moreover, the computational cost of the method we proposed is $I \times J \times P$, and the others are $I \times J \times P \times P$. Obviously, the computational effort of RSONN is significantly reduced.

\section{V.SimULATION STUdies}

In this section, the performance of RSONN is tested over three benchmark tasks and one real world application, specially, they are: two classical nonlinear system modeling problems, the well-known Mackey-Glass time-series prediction problem, and the sludge bulking prediction in wastewater treatment process (WWTP). For simplicity, the initial parameters of the RSONN in these examples are the same: $\eta=0.39, \sigma=0.54, c=0.08, w=0.07, v=0.83$. The results are compared with some other existing methods. All the simulations were programmed with Matlab version 2010, and were run on a PC with a clock speed 2.6 GHz and 4 GB RAM, under a Microsoft Windows 8.0 environment.

The performance of the network are measured using the root mean-square-error (RMSE) function defined as

$$
\operatorname{RMSE}(t)=\sqrt{\frac{1}{2 P} \sum_{p=1}^{P}\left(y_{d}(t)-y(t)\right)^{2}}
$$

where $P$ is the total number of the samples, $y(t)$ and $y_{d}(t)$ are the network output and the desired output at time $t$.

\section{A. Mackey-Glass Time-Series Prediction}

In this example, the effectiveness of RSONN is examined by the following Mackey-Glass time-series prediction problem

$$
x(t+1)=(1-a) x(t)+\frac{b x(t-\tau)}{1+x^{10}(t-\tau)},
$$

where $a=0.1, b=0.2, \tau=17$, and the initial condition $x(0)=1.2$. Following [45-49], the prediction model is given by

$$
x(t+p)=\bar{f}(x(t), x(t-6), x(t-12), x(t-18))
$$

500 data points between $t=1$ and 500 are chosen as the training samples; and another 500 data points between $t=501$ and 1000 are given as the testing samples. The initial structure of RSONN is 4-3-1, 4 neurons in input layer, 3 neurons in hidden 
layer and 1 neuron in output layer. The initial radius of each hidden neurons is $\sigma=2.5$. The pre-set training RMSE was 0.01 . The results are shown in Figs. 4-7.

Fig. 4 shows the number of the hidden neurons in the training process. And the RMSE values in the training process are illustrated in Fig. 5. Moreover, Fig. 6 displays the predicting results of RSONN, and Fig. 7 shows the predicting error between the desired output and the predicting results. Figs. 6 and 7 show that the proposed RSONN can predict the Mackey-Glass time-series efficiently. A comparison between the proposed RSONN and the other existing algorithms is shown in Table 1.

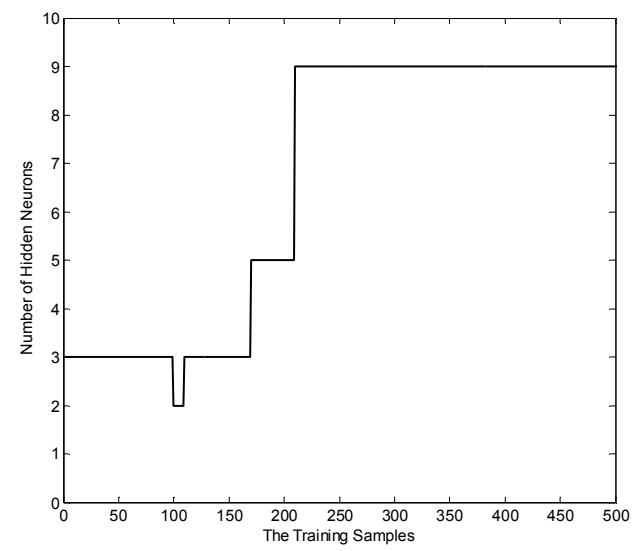

Fig. 4. The number of hidden neurons in the training process

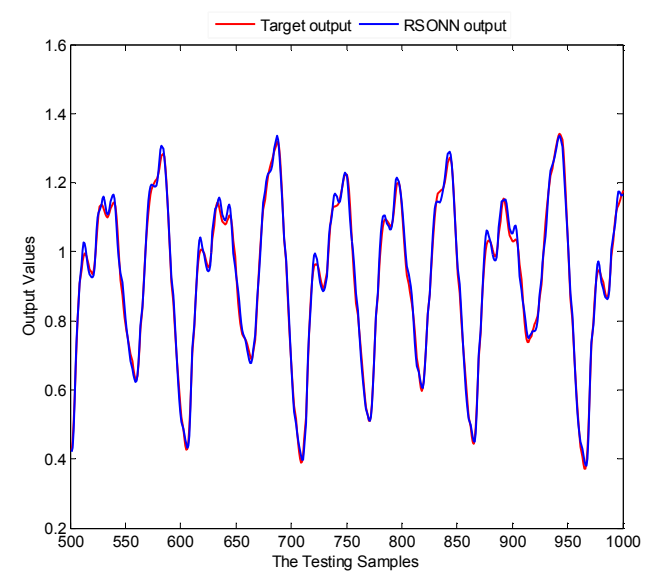

Fig. 6. The predicting results of RSONN

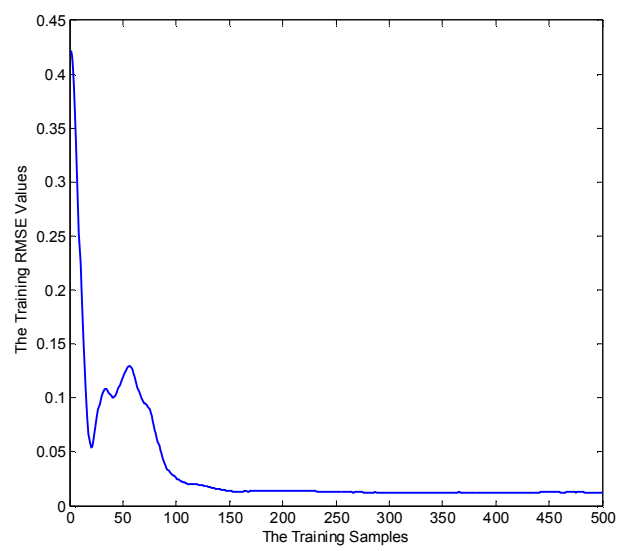

Fig. 5. RMSE values in the training process

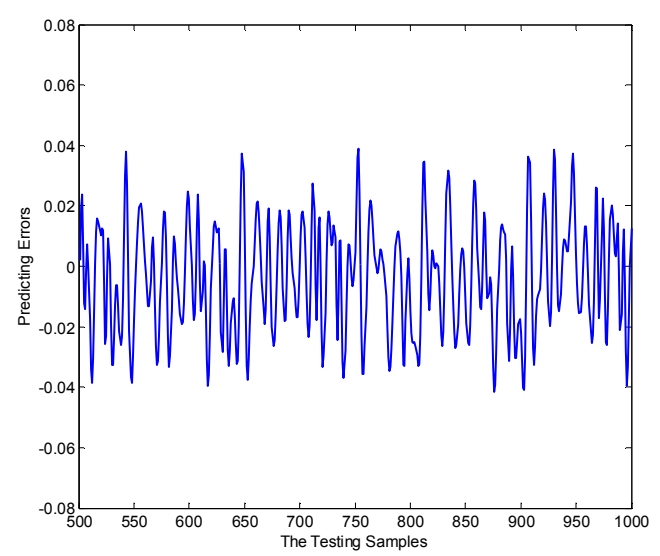

Fig. 7. The predicting errors of RSONN

Table 1: The performance comparison of the different approaches

\begin{tabular}{|c|c|c|c|}
\hline \multirow{2}{*}{ Methods } & \multirow{2}{*}{ Hidden Neurons } & \multicolumn{2}{|c|}{$R M S E$} \\
\hline & & Training RMSE & Testing RMSE \\
\hline RSONN & 9 & 0.01 & 0.0110 \\
\hline TRFN-S [28] & $7 *$ & - & $0.0124^{*}$ \\
\hline RNN [45] & $10 *$ & $0.1183^{*}$ & $0.0946^{*}$ \\
\hline ARN [46] & $17 *$ & $0.039 *$ & $0.0340^{*}$ \\
\hline ERNN [47] & $13 *$ & - & 0.0690 \\
\hline SORBF [48] & $12 *$ & $0.01 *$ & $0.0121^{*}$ \\
\hline MGAP-RBF [48] & $19 *$ & $0.01 *$ & $0.0264^{*}$ \\
\hline SOFMLS [49] & $7 *$ & - & 0.0471 \\
\hline GAP-RBF [49] & $19 *$ & $0.01 *$ & $0.0312 *$ \\
\hline
\end{tabular}

*The results are listed in original papers. 
Table 1 shows the different methods for predicting the Mackey-Glass time series, it consists of two parts. Firstly, the proposed RSONN compared with some existing recurrent neural networks: the recurrent neural network (RNN) [45], the accelerated recurrent network (ARN) [46] and the evolutionary recurrent neural network (ERNN) [47]. Secondly, the proposed RSONN is also compared with some self-organizing neural networks such as the self-organizing RBF (SORBF) neural networks [48], the modified growing and pruning RBF (MGAP-RBF) [48], the self-organizing fuzzy modified least-square (SOFMLS) [49], and the growing and pruning RBF (GAP-RBF) neural networks [49]. It can be seen that the proposed RSONN obtains the most compact structure for predicting the Mackey-Glass time series. Meanwhile, the testing RMSE values (0.0110) of RSONN are the smallest in the experiment. Table 1 also indicates that the predicting values obtained using the proposed RSONN with respect to the real output is more accurate than those obtained from the other algorithms in this example.

\section{B. Henon Chaotic Series Prediction}

The discrete time Henon chaotic system [64] with one delay and two parameters is described as

$$
y(t+1)=-P y^{2}(t)+Q y(t-1)+1.0
$$

where $P=1.4$ and $Q=0.3$. In this example, the input of the RSONN is $y(t-1)$ and the output is $y(t)$. 2000 data points are generated by the initial state is $[y(1), y(0)]=[0.4,0.4]$, where the first 1000 data points were used as the training samples and the remaining 1000 data points were used as the testing samples. The initial structure of RSONN is $2-8-1$. The initial weight of every hidden neuron is chosen from a uniform distribution in the range [0, 1]. The pre-set training RMSE is 0.01 .

The results are presented in Figs. 8-11. Figs. 8-9 show the number of hidden neurons and the RMSE values in the training process, respectively. And the testing results in Figs. 10-11 show that the proposed RSONN can model the nonlinear system efficiently.

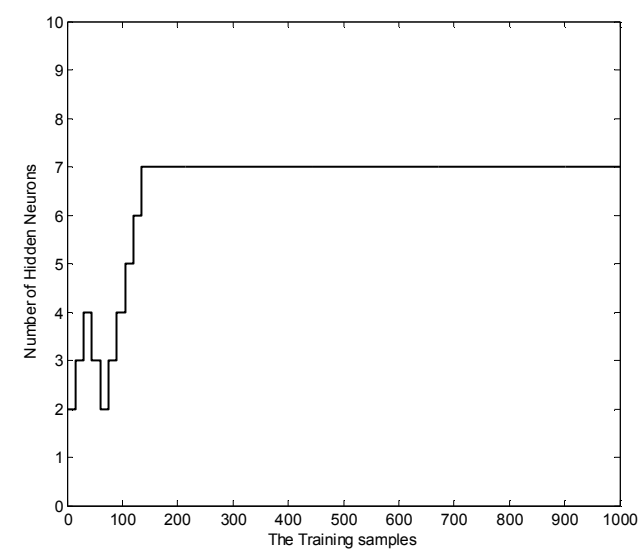

Fig. 8. The number of hidden neurons in the training process

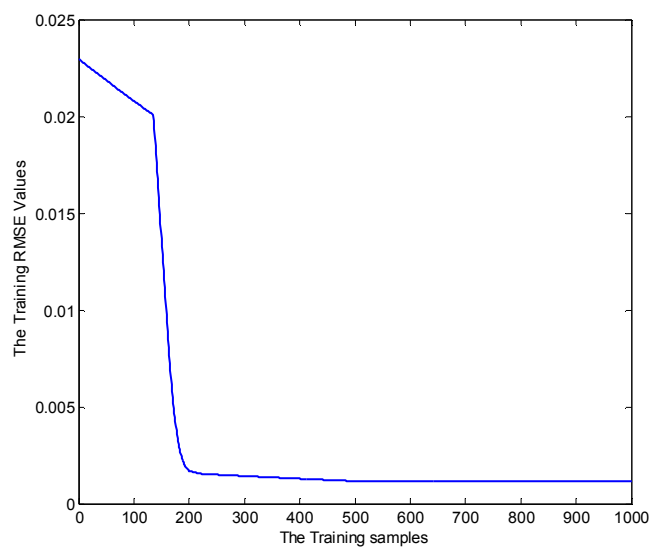

Fig. 9. RMSE values in the training process 


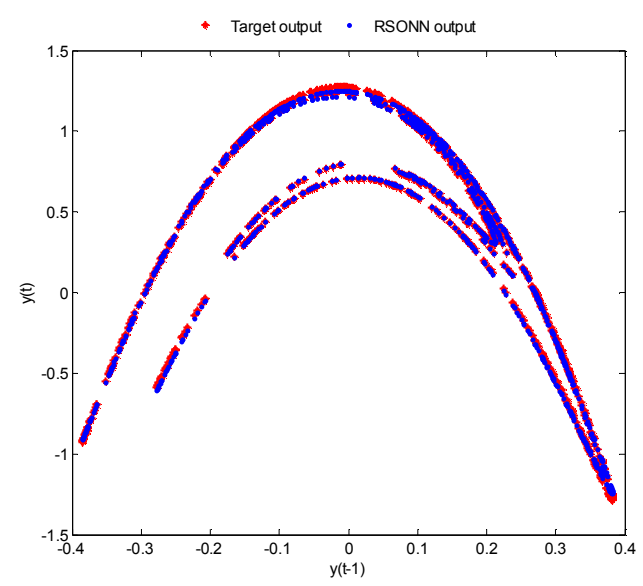

Fig. 10. The predicting results of RSONN

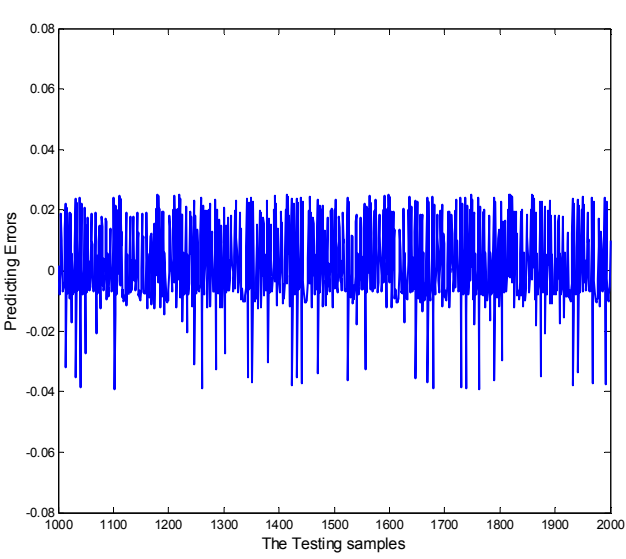

Fig. 11. The predicting errors versus testing samples

Moreover, in order to check the performance of RSONN, a comparison between RSONN and the other existing algorithms: the self-organizing neural networks (RSEFNN-LF [30], IRSFNN [28], MRIT2NFS [32], SOFNN [50]) and the recurrent neural network (RLS-ESN [51], RFNN [52], WRFNN [53]) is presented in Table 2, where the final number of hidden neurons, the running time, the training and testing RMSE are shown in details. It can be seen that the number of hidden neurons in RSONN is 6 which is less than that of the other algorithms (except IRSFNN [28] and MRIT2NFS [32]). The testing RMSE of RSONN is the smallest among these different algorithms. Also, the proposed RSONN needs less CPU time (27.26s) among all algorithms (except RFNN [52]). This details in Table 2 demonstrate that the proposed RSONN has better predicting ability compared to the other algorithms (both self-organizing neural networks and recurrent neural networks).

Table 2: The performance comparison of the different approaches

\begin{tabular}{ccccc}
\hline Algorithms & Hidden Neurons & Running Times (s) & RMSE \\
\cline { 3 - 5 } & & $\mathbf{6}$ & $\mathbf{2 7 . 2 6}$ & Training \\
\hline RSONN & $7^{*}$ & - & $\mathbf{0 . 0 1 2 2}$ \\
\hline RSEFNN-LF [27] & $3^{*}$ & - & $0.0320^{*}$ & $0.016^{*}$ \\
IRSFNN [28] & $2^{*}$ & - & $0.01^{*}$ & $0.0140^{*}$ \\
MRIT2NFS [32] & $10^{*}$ & $27.97^{*}$ & $0.0560^{*}$ \\
SOFNN [50] & $300^{*}$ & $43.21^{*}$ & $0.1974^{*}$ & $0.2021^{*}$ \\
\hline RLS-ESN [51] & 15 & 10.68 & 0.4630 & 0.0130 \\
RFNN [52] & 7 & 23.89 & 0.1910 \\
WRFNN [53]
\end{tabular}

*The results are listed in original papers.

\section{Nonlinear Dynamic System Identification}

The nonlinear dynamic system, which has been used in several papers, notably [54-55], is used to demonstrate the effectiveness of RSONN

$$
y(t+1)=\frac{y(t) y(t-1)[y(t)+2.5]}{1+y^{2}(t)+y^{2}(t-1)}+u(t)
$$

where, $1 \leq t \leq 500, y(0)=0, y(1)=0, u(t)=\sin (2 \pi t / 25)$.

The model is described by the following equation 


$$
y(t+1)=f(y(t), y(t-1), u(t)) .
$$

There are three inputs and one output in this network. The inputs of RSONN are given by $(y(t), y(t+1), u(t))$; the output is given by $y(t+1)$. In the training phase, a set of 400 points were chosen between $t=1$ and 400 . Another 100 input-target points in the interval $[401,500]$ were used as the testing data. The initial structure of RSONN is 3-3-1. The pre-set training error value was 0.01 . The initial weight of every hidden neuron is chosen from a uniform distribution in the range $[0,1]$.

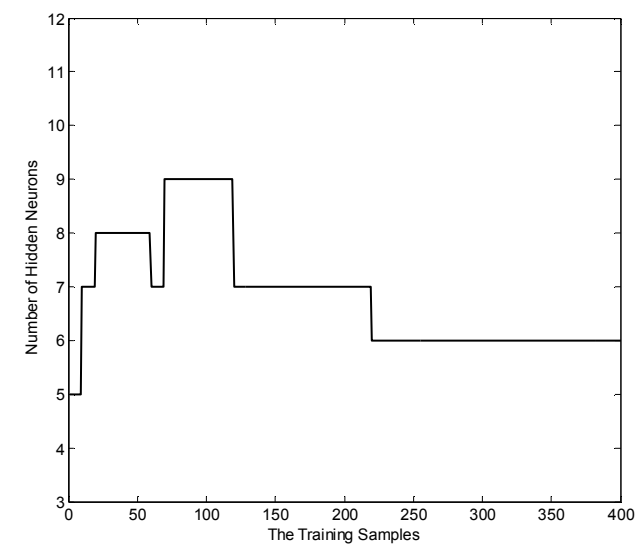

Fig. 12. The number of hidden neurons in the training process

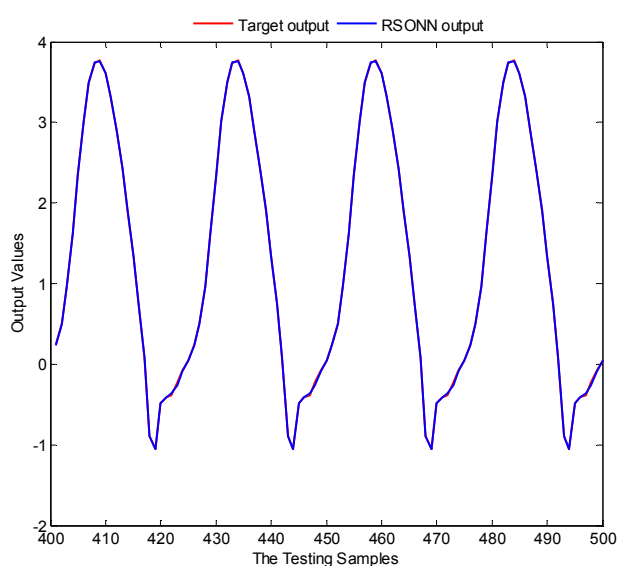

Fig. 14. The predicting results of RSONN

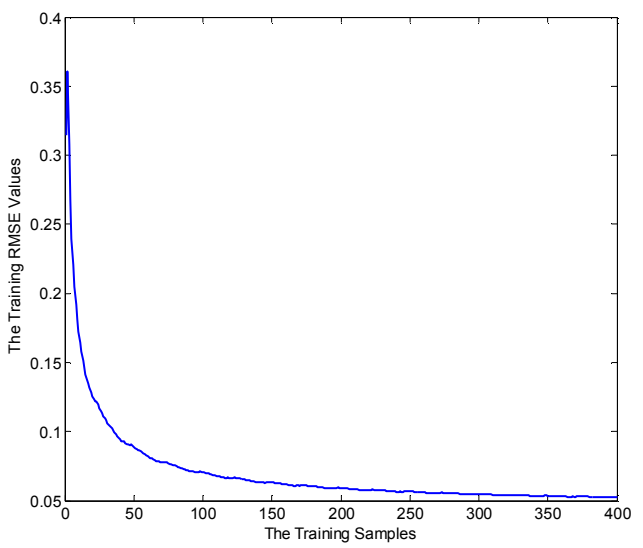

Fig. 13. RMSE values in the training process

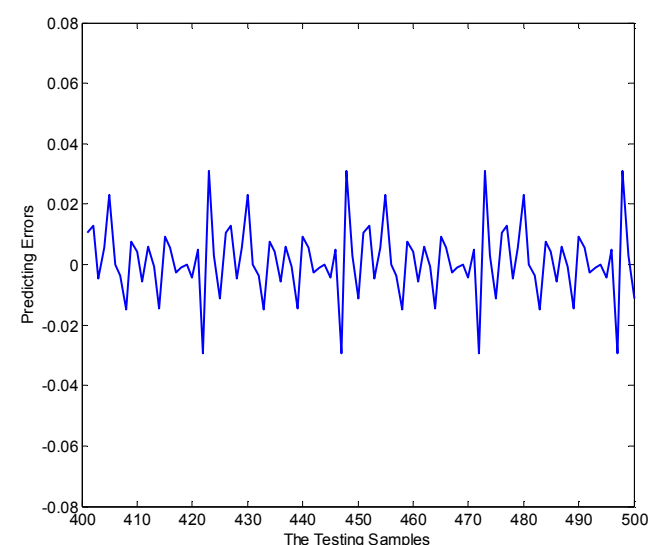

Fig. 15. The predicting errors versus testing samples

Table 3: The performance comparison of the different approaches

\begin{tabular}{ccccc}
\hline Methods & Hidden Neurons & Running Times $(s)$ & RMSE \\
\cline { 3 - 5 } RSONN & $\mathbf{1 1}$ & $\mathbf{1 5 . 8 8}$ & $\mathbf{0 . 0 1}$ \\
\hline GP-FNN[54] & 9 & 16.14 & 0.01 & 0.01 \\
LRFNN-SVR [54] & 3 & 72.10 & 0.008 \\
GDFNN[55] & 11 & 87.12 & - & 0.0107 \\
FAOS-PFNN[56] & 11 & 18.18 & - & 0.0550 \\
OLS[56] & 65 & - & - & 0.0252 \\
RBF-AFS[56] & 35 & - & 0.0288 & 0.1384 \\
FS-RBFNN[48] & $12 *$ & $8.14^{*}$ & $0.0121^{*}$ \\
\hline
\end{tabular}

*The results are listed in original papers. 
Figs. 12-13 show the number of hidden neurons and the RMSE values in training process. The predicting values and the predicting errors are given in Figs.14-15. Meanwhile, the results of RSONN were compared with GP-FNN [54], LRFNN-SVR [54], GDFNN [55], FAOS-PFNN [56], OLS [56], RBF-AFS [56], and FS-RBFNN [48]. All the algorithms have the same training data sets as well as testing partitions. Table 3 shows that the proposed RSONN owns a more compact structure (except GP-FNN and LRFNN-SVR). Moreover, the testing RMSE of the network (0.0084) is less than the other algorithms. The CPU time of the network is 15.88 , which is also shorter than the other algorithms (except FS-RBFNN [48]). This example shows that the proposed RSONN has better predicting ability than all the other existing algorithms.

\section{Sludge Bulking Prediction}

The activated sludge process is the most commonly applied technology for wastewater treatment processes (WWTPs) [57]-[58]. However, in the activated sludge process usually associated with sludge bulking. Despite extensive research that has been done on sludge bulking, it remains a widespread problem in the operation of activated sludge processes, which brings severe economic and environmental consequences. Therefore, the sludge bulking prediction is essential for the wastewater treatment system. In this section, the main objective is to develop a sludge bulking prediction model that provides accurate predictions of the sludge bulking index (SVI) values in WWTPs. In the experiment, the most important water quality parameters such as chemical oxygen demand (COD), biochemical oxygen demand (BOD), influent flow rate (Qin), dissolved oxygen (DO), acidity and basicity $(\mathrm{PH})$, total nutrients $(\mathrm{TN})$ [60], were selected as the input research variables for predicting the SVI values. The input-output water quality data from a real wastewater treatment plant (Beijing, China). After deleting abnormal samples, 1050 samples were obtained and normalized, 450 samples from September to October were used as testing data, whilst the remaining samples were used as training data. The initial structure of the RSONN is 6-3-1. The error measures for the SVI are $15 \mathrm{mg} / \mathrm{L}$ confidence limits.

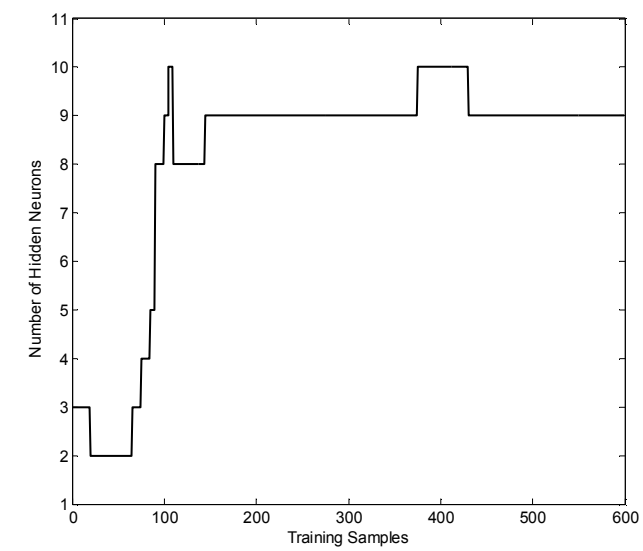

Fig. 16. The number of hidden neurons in the training process

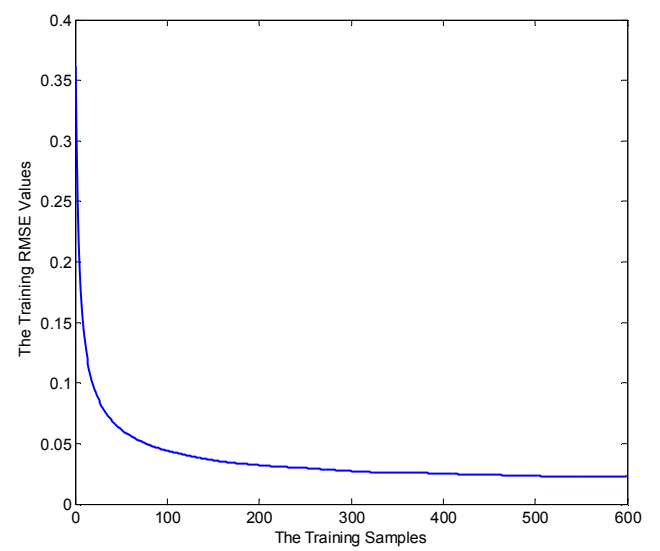

Fig. 17. The RMSE values in the training process 


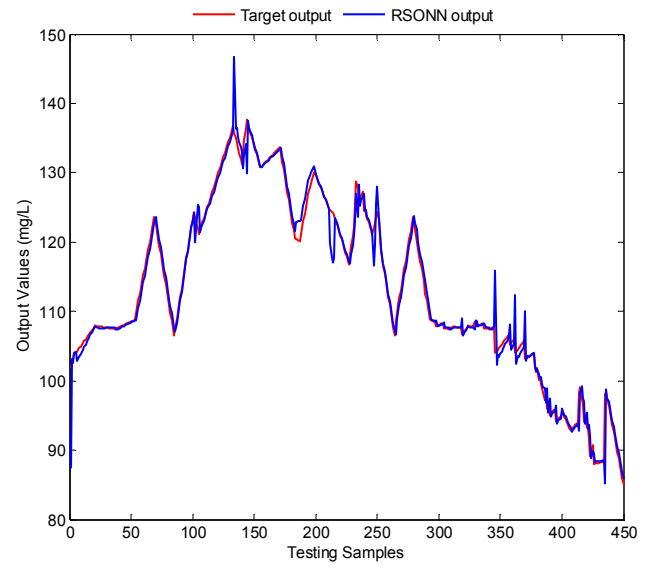

Fig. 18. The predicting SVI values

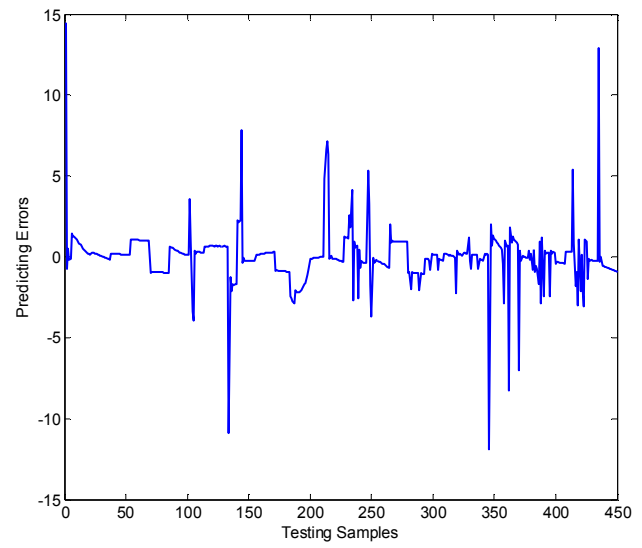

Fig. 19. The predicting errors

The number of hidden neurons in the training process is shown in Fig. 16. Fig. 17 describes the RMSE values in the training process. Fig. 18 shows the predicting results of SVI. And the predicting errors, which are less than $\pm 15 \mathrm{mg} / \mathrm{L}$, is shown in Fig. 19. Additionally, to show the performance of RSONN clearly, the results of RSONN are compared with other methods-SORBF [59], FNN [60], ESRNN [22], RRSEFNN [38], the mathematic method [61], the dynamic ARX method [62], and the Image analysis [63]. In Table 4, the testing accuracy, the mean and deviation (Dev.) of testing RMSE are shown in details.

Table 4: A comparison performance of different methods

\begin{tabular}{|c|c|c|c|c|c|}
\hline \multirow{2}{*}{ Methods } & \multirow{2}{*}{ Hidden neurons } & \multicolumn{2}{|c|}{ Testing RMSE } & \multicolumn{2}{|c|}{ Testing accuracy (\%) } \\
\hline & & Mean & $D e v$ & Min & Mean \\
\hline RSONN & $9 \pm 1$ & 0.238 & 0.000544 & 93.56 & 95.47 \\
\hline SORBF [59] & $13 \pm 1 *$ & 0.731 & 0.001303 & $91.12^{*}$ & $92.46^{*}$ \\
\hline FNN [60] & $16 \pm 1 *$ & 0.533 & 0.000911 & $92.75^{*}$ & $94.38 *$ \\
\hline ESRNN [22] & $10 \pm 1$ & 0.371 & 0.000872 & 81.10 & 88.50 \\
\hline RRSEFNN [38] & $9 \pm 2$ & 0.337 & 0.000752 & 92.54 & 87.78 \\
\hline Mathematic model [61] & - & - & - & $86.00 \%$ & $90.87 \% *$ \\
\hline Dynamic ARX [62] & - & - & - & $77.63 \% *$ & $84.90 \% *$ \\
\hline Image analysis [63] & - & - & - & $84 \%$ & $89 \%$ \\
\hline
\end{tabular}

*The results are listed in original papers.

From Table 4, it can reach the following conclusion: 1) the proposed RSONN owns a more compact structure than the other algorithms, 2) the testing RMSE (the mean and Dev.) of the proposed RSONN is the smallest, 3) the accuracy of the proposed RSONN is better than that of the other methods (other neural network methods and prediction models). Hence, the comparisons demonstrate that this proposed RSONN method is more suitable for the SVI prediction.

\section{CONCLUSION}

In this paper, an AGPA and an adaptive second-order algorithm are proposed to design RNN for modeling uncertain nonlinear system. The proposed RSONN can determine the network size and parameters simultaneously to enhance its generalization capabilities. The effectiveness of RSONN has been confirmed by some experimental results. The results show that the learning efficiency and performance of RSONN are better than a number of other algorithms commonly used in the 
literature. The main contributions of RSONN are as follows: 1) Based on the information processing ability and competitiveness of hidden neurons, the self-organizing mechanism can add or prune the hidden neurons to reduce the computational complexity and improve the generalization performance. 2) The adaptive second-order algorithm with adaptive learning rate can be computed directly to significant memory reduction and speed benefit. 3) The convergence of RSONN has been demonstrated theoretically and experimentally.

In addition, the results in Tables 1-4 indicate that the proposed RSONN achieved better testing RMSE and running times than the other algorithms. Moreover, for sludge bulking prediction, the proposed RSONN is better at predicting sludge bulking than the other methods in WWTPs, which is essential to develop an efficient model prediction controller according to the prediction results.

\section{REFERENCES}

[1] H.Q. Zhao, S.B. Gao, Z.Y. He, X.P. Zeng, W.D. Jin, T.R. Li, Identification of nonlinear dynamic system using a novel recurrent wavelet neural network based on the pipelined architecture, IEEE Transactions on Industrial Electronics 61(8) (2014) 4171-4182.

[2] R. Adhikari, A neural network based linear ensemble framework for time series forecasting, Neurocomputing 157 (1) (2015) 231-242.

[3] K. Li, J. X. Peng, E. W. Bai, A two-stage algorithm for identification of nonlinear dynamic systems, Automatica, 42 (7) (2006) 1189-1197.

[4] G.D. Wu, Z.W. Zhu, An enhanced discriminability recurrent fuzzy neural network for temporal classification problems, Fuzzy Sets and Systems 237(16) (2014) 47-62.

[5] M.A. Escalona-Moran, M.C. Soriano, I. Fischer, C.R. Mirasso, Electrocardiogram classification using reservoir computing with logistic regression, IEEE Journal of Biomedical and Health Informatics 19(3) (2015) 892-898.

[6] C. Smith, Y.C. Jin, Evolutionary multi-objective generation of recurrent neural network ensembles for time series prediction, Neurocomputing 143(2) (2014) 302-311.

[7] E. Egrioglu, U. Yolcu, C.H. Aladag, E. Bas, Recurrent multiplicative neuron model artificial neural network for non-linear time series forecasting, Procedia-Social and Behavioral Sciences 109(8) ( 2014 ) 1094-1100.

[8] C.Y. Sheng, J. Zhao, W. Wang, H. Leung, Prediction intervals for a noisy nonlinear time series based on a bootstrapping reservoir computing network ensemble, IEEE Transactions on Neural Networks and Learning Systems 24(7) (2013) 1036 $-1048$.

[9] R. Zemouri, D. Racoceanu, N. Zerhouni, Recurrent radial basis function network for time-series prediction, Engineering Applications of Artificial Intelligence 16(5-6) (2003) 453-463.

[10]R. Zemouri, J. M. Faure, Comparative study between the timed automata and the recurrent radial basis function for discrete event system diagnosis, IFAC Proceedings Volumes 39(13) (2003): 1455-1460.

[11]X. Tong, Z. Wang, H. Yu, A research using hybrid RBF/Elman neural networks for intrusion detection system secure model, Computer physics communications 180(10) (2009): 1795-1801.

[12] M. Fairbank, S. Li, X. Fu, E. Alonso, D. Wunsch, An adaptive recurrent neural-network controller using a stabilization matrix and predictive inputs to solve a tracking problem under disturbances, Neural Networks 49(1) (2014): 74-86.

[13] M. C. Phan, M. T. Hagan. Error surface of recurrent neural networks, IEEE Transactions on Neural Networks and Learning Systems 24(11) (2013): 1709-1721.

[14]F.F.M El-Sousy, Adaptive dynamic sliding-mode control system using recurrent RBFN for high-performance induction motor servo drive, IEEE Transactions on Industrial Informatics 9(4) (2013): 1922-1936.

[15]F.J. Lin, P.H. Shen, S.L. Yang, P.H. Chou, Recurrent radial basis function network-based fuzzy neural network control for permanent-magnet linear synchronous motor servo drive, IEEE Transactions on Magnetics 42(11) (2006): 3694-3705.

[16]C.F. Juang, C.D. Hsieh, Recurrent fuzzy neural network with support vector regression for dynamic-system modeling, IEEE Transactions on Fuzzy Systems 18(2) (2010) 261-273. 
[17]X.X. Wang, L.Y. Ma, B.S. Wang, T. Wang, A hybrid optimization-based recurrent neural network for real-time data prediction, Neurocomputing 120 (2013) 547-559.

[18] M. Tofighi, M. Alizadeh, S. Ganjefar, M. Alizadeh, Direct adaptive power system stabilizer design using fuzzy wavelet neural network with self-recurrent consequent part, Applied Soft Computing 28(1) (2015) 514-526.

[19]F.F.M. El-Sousya, Adaptive hybrid control system using a recurrent RBFN-based self-evolving fuzzy-neural-network for PMSM servo drives, Applied Soft Computing 21(1) (2014) 509-532.

[20] M.B. Hastings, G.H. Watson, R.G. Melko, Self-correcting quantum memories beyond the percolation threshold, Physical Review 112(7-21) (2014) 070501.

[21]D.T. Mirikitani, N. Nikolaev, Recursive bayesian recurrent neural networks for time-series modeling, IEEE Transaction on Neural Networks 21(2) (2010) 262-274.

[22]J.Yin, Y. Meng, Y.C. Jin, A developmental approach to structural self-organization in reservoir computing, IEEE Transaction on Autonomous Mental Development 4(4) (2012) 273 - 289.

[23]C.S. Leung, A.C. Tsoi, Combined learning and pruning for recurrent radial basis function networks based on recursive least square algorithms, Neural Comput \& Applic 15(1) (2005) 62-78.

[24]A.M. Rather, A. Agarwal, V.N. Sastry, Recurrent neural network and a hybrid model for prediction of stock returns, Expert Systems with Applications 42 (2015) 3234-3241.

[25] S. Kumar, S. Kumar, Prakash, R. Shankar, M.K. Tiwari, S.B. Kumar, Prediction of flow stress for carbon steels using recurrent self-organizing neuro fuzzy networks, Expert Systems with Applications 32(3) (2007) 777-788.

[26] J.K. Liu, D.V. Buonomano, Embedding multiple trajectories in simulated recurrent neural networks in a self-organizing manner, The Journal of Neuroscience 29(42) (2009) 13172-13181.

[27]N. Subrahmanya, Y.C. Shin, Constructive training of recurrent neural networks using hybrid optimization, Neurocomputing 73(13-15) (2010) 2624-2631.

[28] Y.Y. Lin, J.Y. Chang, C.T. Lin, Identification and prediction of dynamic systems using an interactively recurrent self-evolving fuzzy neural network, IEEE Transactions on Neural Networks and Learning Systems 24(2) (2013) $310-321$.

[29]C.F. Hsu, Intelligent position tracking control for LCM drive using stable online self-constructing recurrent neural network controller with bound architecture, Control Engineering Practice 17(6) (2009) 714-722.

[30]C.F. Juang, Y.Y. Lin, C.C. Tu, A recurrent self-evolving fuzzy neural network with local feedbacks and its application to dynamic system processing[J]. Fuzzy Sets and Systems, 2010, 161(19): 2552-2568.

[31]F.F.M. El-Sousya, Adaptive hybrid control system using a recurrent RBFN-based self-evolving fuzzy-neural-network for PMSM servo drives, Applied Soft Computing 21(1) (2014) 509-532.

[32] Y.Y. Lin, J.Y. Chang, N.R. Pal, C.T. Lin, A mutually recurrent interval type-2 neural fuzzy system (MRIT2NFS) with self-evolving structure and parameters, IEEE Transactions on Fuzzy Systems 21(3) (2013) 492-509.

[33]J. Iglesias, A.E.P. Villa, Recurrent spatiotemporal firing patterns in large spiking neural networks with ontogenetic and epigenetic processes, Journal of Physiology-Paris 104(3-4) (2010) 137-146.

[34]H.S. Wang, X.F. Yan, Improved simple deterministically constructed cycle reservoir network with sensitive iterative pruning algorithm, Neurocomputing 145(1) (2014) 353-362.

[35]A. Garliauskas, Self-organized topological structures in neural networks for the visual cortex of the brain, Neurocomputing 51 (2003) 13-39.

[36]C.F. Juang, R.B. Huang, Y.Y. Lin, A recurrent self-evolving interval type-2 fuzzy neural network for dynamic system processing, IEEE Transactions on Fuzzy Systems 17(5) (2009) 1092-1105.

[37]C.S. Chen, TSK-type self-organizing recurrent-neural-fuzzy control of linear microstepping motor drives, IEEE Transactions on Power Electronics 25(9) (2010) 2253-2265.

[38]C.F. Hsua, K.H. Cheng, Recurrent fuzzy-neural approach for nonlinear control using dynamic structure learning scheme, Neurocomputing 71(16-18) (2008) 3447-3459.

[39]T. Aoki, Self-organization of a recurrent network under ongoing synaptic Plasticity, Neural Networks 62(1) (2015) 11-19.

[40] J. Eser, P.S. Zheng, J. Triesch, Nonlinear dynamics analysis of a self-organizing recurrent neural Network: Chaos Waning, PloS One 9(1) (2014) e86962.

[41]H.G. Han, L.M. Ge, J.F. Qiao, An adaptive second order fuzzy neural network for nonlinear system modeling, Neurocomputing 214(1) (2016) 837-847.

[42]L. Luo, D.D.M. O'Leary, Axon retraction and degeneration in development and disease, Annual Review of Neuroscience 28(1) (2005) 127-156.

[43]N. Ampazis, S.J. Perantonis, Two highly efficient second-order algorithms for training feedforward networks, IEEE Transactions on Neural Networks 13(5) (2002) 1064-1074.

[44]C.H. Lu, Wavelet fuzzy neural networks for identification and predictive control of dynamic systems, IEEE Transactions on Industrial Electronics 58(7) (2011) 3046-3058.

[45] T.W.S. Chow, S.Y. Cho, An accelerated recurrent network training algorithm using IIR Filter model and recursive least squares method, IEEE Transactions on Circuits and System-I: Fundamental Theory and Applications 44(11) (1997) 
1092-1086.

[46]R.V. Kulkarni, G.K. Venayagamoorthy, Generalized neuron: feedforward and recurrent architectures, Neural Networks 22(7) (2009) 1011-1017.

[47]R. Chandra, M.J. Zhang, Cooperative coevolution of Elman recurrent neural networks for chaotic time series prediction, Neurocomputing 86(1) (2012) 116-123.

[48]H.G. Han, Q.L. Chen, J.F. Qiao, An efficient self-organizing RBF neural network for water quality prediction, Neural Networks 24(7) (2011) 717-725.

[49] G.B. Huang, P. Saratchandran, N. Sundarajan, An efficient sequential learning algorithm for growing and pruning RBF (GAP-RBF) networks, IEEE Transactions on Neural systems, man, and cybernetics-part B: cybernetics 34(6) (2011) 2284-2292.

[50]C.F. Juang, C.D. Hsieh, A locally recurrent fuzzy neural network with support vector regression for dynamic-system modeling, IEEE Transactions on Fuzzy Systems, 18(2) (2010) 261-273.

[51]H. Jaeger, Adaptive nonlinear system identification with echo state networks, Advances in neural information processing process (2002) 593-600.

[52] C.H. Lee, C.C. Teng, Identification and control of dynamic systems using recurrent fuzzy neural networks, IEEE Transactions on Fuzzy Systems, 8 (4) (2000) 349-366.

[53]C.J. Lin, C.C. Chin, Prediction and identification using wavelet-based recurrent fuzzy neural networks, IEEE Transactions on Systems, Man, and Cybernetics 34 (5) (2004) 2144-2154.

[54]H.G. Han, J.F. Qiao, A self-organizing fuzzy neural network based on a growing-and-pruning algorithm, IEEE Transactions on Fuzzy Systems 18(6) (2010) 1129-1143.

[55] S. Wu, M.J. Er, Y. Gao, A fast approach for automatic generation of fuzzy rules by generalized dynamic fuzzy neural networks, IEEE Transactions on Fuzzy Systems 9(4) (2001) 578-594.

[56]N. Wang, M.J. Er, X. Meng, A fast and accurate online self-organizing scheme for parsimonious fuzzy neural networks, Neurocomputing, 72(16) (2009) 3818-3829.

[57] O. Gustaf, ICA and me -A subjective review, Water Research 46(6) (2012) 1585-1624.

[58]J.M. Brault, R.L. Labib, M. Perrier, P. Stuart, Prediction of activated sludge filamentous bulking using ATP data and neural networks, Canadian Society for Chemical Engineering 86(5) (2011) 901-913.

[59]H.G. Han, J.F. Qiao, Prediction of activated sludge bulking based on a self-organizing RBF neural network, Journal of Process Control 22(6) (2012) 1103-1112.

[60]H.G. Han, Y. Li, J.F. Qiao, A fuzzy neural network approach for online fault detection in waste water treatment process, Computers \& Electrical Engineering 40(7) (2014) 2216-2226.

[61]D.L. Giokas, G.T. Daigger, M. Sperling, Y. Kim, P.A. Paraskevas, Comparison and evaluation of empirical zone settling velocity parameters based on sludge volume index using a unified settling characteristics database, Water Research 37(16) (2003) 3821-3836.

[62]I.Y. Smets, E.N. Banadda, J. Deurinck, N. Renders, R. Jenne, J.F.V. Impe, Dynamic modeling of filamentous bulking in lab-scale activated sludge processes, Journal of Process Control 16(3) (2006) 313-319.

[63]D.P. Mesquita, O. Dias, A.A.M. Dias, A.L. Amaral, E.C. Ferreira, Correlation between sludge settling ability and image analysis information using partial least squares, Analytica chimica acta 642(1) (2009) 94-101.

[64]C.K.D Santos, R.P. Espíndola, V.F. Vieira, A.G. Evsukoff, Identification of dynamic systems Using a differential evolution-based recurrent fuzzy system, 2014 IEEE International Conference on Fuzzy Systems (FUZZ-IEEE) 1691-1696. 